\title{
TOWARDS AEROTHERMOELASTIC SIMULATIONS OF SUPERSONIC FLOW THROUGH NOZZLES
}

\author{
N. Hosters ${ }^{1}$, M. Klaus ${ }^{2}$, G. Schieffer ${ }^{1}$, M. Behr ${ }^{1}$, \\ and H.-G. Reimerdes ${ }^{2}$ \\ ${ }^{1}$ RWTH Aachen University \\ 2 Schinkelstr., Aachen 52062, Germany \\ ${ }^{2}$ Department of Aerospace and Lightweight Structures \\ RWTH Aachen University \\ 7 Wüllnerstr., Aachen 52062, Germany
}

\begin{abstract}
Efficient numerical methods are necessary to simulate the interaction of aerodynamic loading, heat transfer, and structural deformation. This paper presents a partitioned approach using seperate single field solvers for fluid and thermoelastic problems coupled by the third software package. The methods implemented in the used modules are described. On the way towards an aerothermoelastic solver, a stepwise validation is required. Here, the successful validation of the thermoelastic and aeroelastic solver is shown.
\end{abstract}

\section{INTRODUCTION}

Spacecraft nozzles are exposed to extreme aerodynamic and thermal loads during the launch and the ascent stages. Within the atmosphere, the nozzle flow is mostly overexpanded, so that shock waves occur inside the nozzle, which may lead to restricted shock separation or free shock separation. Asymmetries of these shock-detachment phenomena in circumferential direction cause deformations of the nozzle wall which, in turn, have an influence on the shock detachment itself. Additionally, the nozzle deformation can be affected by the exterior flow. Even if the side loads do not result in a structural failure, the induced vibrations represent a considerable loading of the nozzle and the connected structural components. If such dynamical loads are not considered sufficiently during the design stage, the rocket engine can be damaged.

A fast and reliable simulation tool is useful to estimate the behavior of a nozzle in all flight stages. The computation of the unsteady aerothermoelastic effects applying complex models is very expensive and not practicable in early

This is an Open Access article distributed under the terms of the Creative Commons Attribution License 2.0, which permits unrestricted use, distribution, and reproduction in any medium, provided the original work is properly cited. 
design stages. The application of reduced structural models can decrease the computational costs to support the design of aerothermoelastically optimized nozzles. Furthermore, a partitioned approach by coupling a thermoelastic and a fluid solver can reduce the effort in software development.

Following these needs, the long-term objective of this work is to develop a comprehensive solution method for nonstationary aerothermoelastic problems of structures exposed to high-enthalpy flows. In this paper, the implemented methods and first simulation results are presented.

\section{NUMERICAL METHODS}

The solver applied here follows a partitioned approach. In this approach, separate programs are operated iteratively to solve the coupled four-field problem. This problem consists of the heat conduction and its influence on the structural deformation as well as the combined interaction of temperature and deformation with the unsteady flow field, which is solved on a deforming grid.

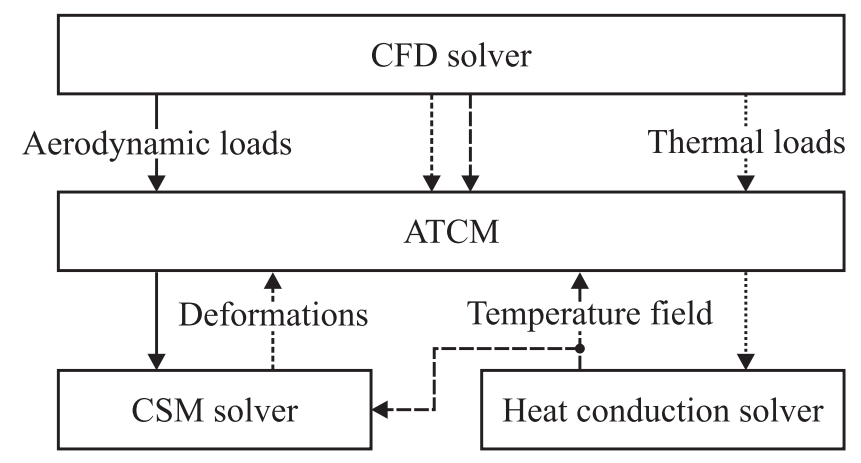

Figure 1 Scheme of the aerothermoelastic coupling chain (CSM - computational structural mechanics)

Figure 1 shows a flow chart of the realization of the partitioned numerical approach. Based on this scheme, the computational fluid dynamics (CFD) solver QUADFLOW is coupled with the thermoelastic solver ASTRA by the Aero-Thermoelastic Coupling Module (ATCM) which performs the information between the single field solvers. The individual programs are described in the following.

\subsection{QUADFLOW}

The flow solver QUADFLOW has been developed within the Collaborative Research Center SFB 401 Flow Modulation and Fluid-Structure Interaction over 
the last decade [1]. It solves the compressible versions of the two- or threedimensional Euler- and Reynolds-averaged Navier-Stokes equations. These are discretised with a cell-centered finite volume (FV) method. QUADFLOW features a highly reliable and efficient local grid refinement method based on multiscale analysis [2]. QUADFLOW represents the grid as an overall multiblock topology in which the grid coordinates of each block are computed by evaluating parametric B-spline mappings. The adaptation produces hierarchically nested quadtree-, respectively, octree-type refinements of favored quadrilateral, respectively, hexahedral cells. In the course of fluid-structure interaction (FSI) iterations, the B-spline grid is deformed in QUADFLOW in two steps [3]. At first, the displacements of the multiblock topology are interpolated from the surface deformation with radial basis functions. Afterwards, the B-spline control points within each block are moved by transfinite interpolation.

\section{$2.2 \quad$ ASTRA}

The ASTRA code was developed for static and stability analysis by the Department of Aerospace and Lightweight Structures at RWTH Aachen University. It allows the structural analysis of shells of revolution with an arbitrary meridian. Although this means that the undeformed structure has to be circular in circumference, the loads and the response to them can be arbitrary. A variety of different wall designs is considered, from isotropic to anisotropic formulations. Also, common stiffened shell structures (frame/stringer, waffle-grid, isogrid) can be approximated using smeared properties. Ring-stiffeners are modeled as discrete structures. Due to the use of reduced models which employ the analytical solution of the shell differential equations, ASTRA is an efficient alternative to complete three-dimensional (3D) solvers. The elastic module is extended and a new thermoelastic module is developed to make ASTRA a fast and efficient tool to compute the structural responses to transient mechanical and thermal loadings.

\subsubsection{Elastic Module (ASTRA)}

The elements used in this program are based on a complete shell bending theory [4]. Shear-rigid and shear-deformable elements are implemented in the code.

The ASTRA models are idealized as one-dimensional line elements with four degrees of freedom (three displacement $\left(v_{\xi}, v_{\psi}\right.$, and $\left.v_{\zeta}\right)$ plus the tangential rotation $\left(\varphi_{\xi}\right)$ ). The shear-deformable elements have additionally the rotation along the axial direction $\left(\varphi_{\psi}\right)$ as a fifth degree of freedom. The mechanical formulations for axisymmetric shells in ASTRA describe the stress/deformation field due to external loads and temperature fields, which have a linear temperature distribution over the wall thickness. 


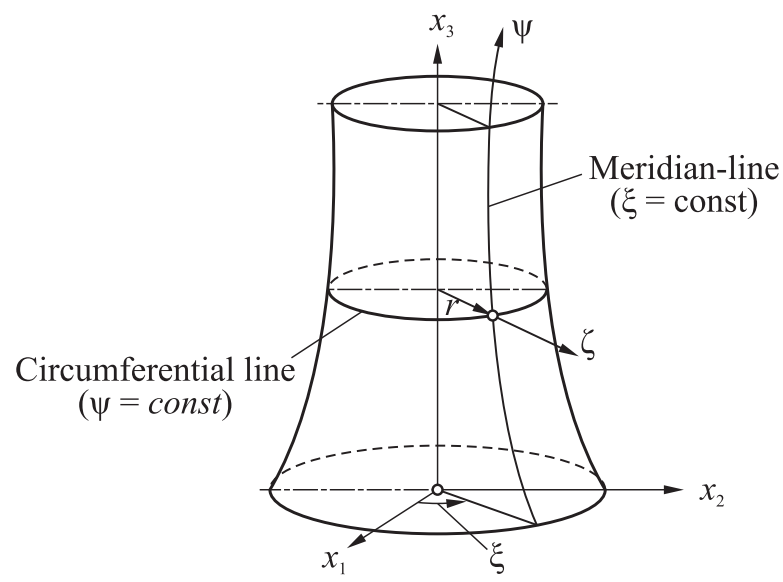

Figure 2 Generic axisymmetric body

The theoretical description of the shell of revolution leads to a system of partial differential equations. The system of equations is transformed into ordinary differential equations using Fourier series in the circumferential direction:

$$
\frac{\partial}{\partial \psi} \vec{Z}(\xi, \psi)=\mathbf{A} \vec{Z}(\xi, \psi)+\vec{L}(\xi, \psi, \vec{\theta})
$$

where $\vec{Z}$ is the state variables (deformations + internal loads); $\mathbf{A}$ is the the differential matrix; $\vec{L}$ are the external loads; $\xi$ is the circumferential direction (Fig. 2); $\psi$ is the meridional direction (see Fig. 2); and $\vec{\theta}$ are the temperatures.

The differential matrix $\mathbf{A}$ is numerically integrated in the meridional direction using a Runge-Kutta method. By rearranging the resulting integral matrix, the element stiffness matrix is obtained.

The loads and the deformations can be arbitrarily distributed in circumferential direction and are expressed by Fourier series (Fig. 3). The following

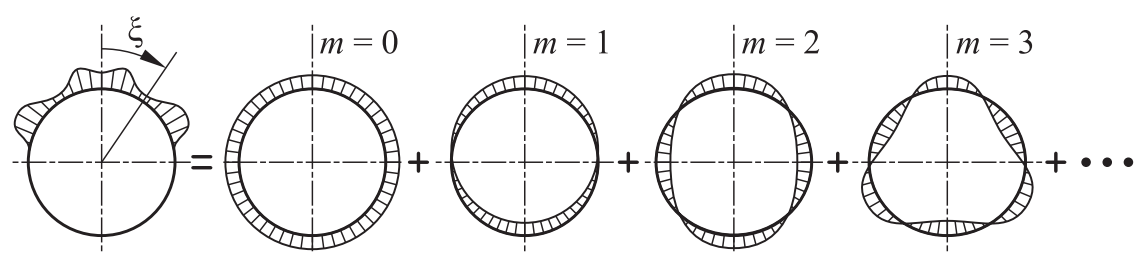

Figure 3 Superposition of waveforms to achieve an arbitrary circumferential distribution 
methods for the structural analysis are implemented in ASTRA: linear static analysis (first-order theory); second-order theory static analysis; fully nonlinear static analysis; linear stability analysis; nonlinear stability analysis, and linear stability analysis with combined loads $[5,6]$.

Also for the investigation of the structural response due to transient aerodynamic and thermoelastic loads, a dynamic module is developed. This is the first step to allow coupled transient analysis with a flow solver via the parallel developed ATCM module. As time integration method the implicit Newmark procedure is implemented.

\subsubsection{Thermoelastic Module (ASTRA)}

To couple ASTRA with the aerothermoelastic coupling module ATCM, the transient thermal loads from the fluid solver, like thermal fluxes, have to be processed and incorporated into the analysis. The thermoelastic analysis with a weak coupling between the thermal and the elastic solutions is assumed (the thermal field is used to compute the deformation field, but not otherwise). The small errors compared to the fully coupled computations are accepted to simplify the problem and, consequently, reduce the computational effort.

$$
\begin{aligned}
\mathbf{M} \ddot{\vec{U}}(t)+\mathbf{K} \vec{U}(t)+\mathbf{\Omega} \vec{\theta}(t) & =\vec{F}(t) \\
\mathbf{M}_{T} \dot{\vec{\theta}}(t)+\mathbf{K}_{T} \vec{\theta}(t) & =\vec{F}_{T}(t)
\end{aligned}
$$

where $\boldsymbol{\Omega}$ is the thermoelastisity; $\mathbf{M}_{T}$ is the thermal capacity; $\mathbf{K}_{T}$ is the thermal conductivity; $\vec{U}$ is the deformation field; $\vec{\theta}(t)$ is the temperature filed; and $\vec{F}(t)$ and $\vec{F}_{T}(t)$ are the mechanical and thermal loads, respectively.

The computed temperature field along with the deformation state is transformed back to the fluid solver via the ATCM.

The heat conduction equation for the axisymmetric shells is given as

$$
\frac{\partial \theta}{\partial t}-\frac{1}{c \rho}\left(\lambda_{2} \frac{\partial r / \partial \psi}{r} \frac{\partial \theta}{\partial \psi}+\lambda_{2} \frac{\partial^{2} \theta}{\partial \psi^{2}}+\lambda_{1} \frac{1}{r^{2}} \frac{\partial^{2} \theta}{\partial \xi^{2}}+\lambda_{3} \frac{\partial^{2} \theta}{\partial \zeta^{2}}\right)-\frac{\eta}{c \rho}=0
$$

where $\zeta$ is the shell normal direction; $\lambda_{1}, \lambda_{2}$, and $\lambda_{3}$ are the coefficients of thermal conductivity in $\xi, \psi$, and $\zeta$ directions; $c$ is the specific heat; $\rho$ is the density; and $\eta$ is the specific heat generation inside the shell.

The thermal fluxes through the inner $\left(q_{i}\right)$ and the outer $\left(q_{a}\right)$ surface of the shell are defined as the temperature gradients at these positions $(\zeta= \pm h / 2$, with $h$ as the local shell thickness): 


$$
\begin{gathered}
\lambda_{3}\left[\frac{\partial T}{\partial \zeta}\right]_{\zeta=-h / 2}=q_{i} ; \\
\lambda_{3}\left[\frac{\partial T}{\partial \zeta}\right]_{\zeta=h / 2}=q_{a} .
\end{gathered}
$$

The temperature field through the thickness is assumed to be quadratic:

$$
\theta(\xi, \psi, \zeta, t)=T_{0}(\xi, \psi, t)+T_{1}(\xi, \psi, t) \zeta+T_{2}(\xi, \psi, t) \zeta^{2} ;
$$

so, the thermal field can be described using three degrees of freedom $\left(T_{0}, T_{1}\right.$, and $T_{2}$ ) for each discrete shell position. A quadratic distribution through the thickness is mostly satisfactory for simple wall configurations. To accommodate complex wall geometries, a higher-order polynomial can be easily implemented using the same procedure as described below with additional degrees of freedom $\left(T_{3}\right.$, etc.). Here, a simple (quadratic polynomial) and, consequently, fast model is adopted to minimize the computational time.

Using the assumptions from Eqs. (3)-(5), the heat conduction equation (Eq. (2)) can be integrated through the thickness of the shell. Additionally, the same equation is multiplied with $\zeta$ and also with $\zeta^{2}$ prior to the integration. After the integrations, the set of three equations emerges:

$$
\begin{aligned}
&\left(\dot{T}_{0}+\dot{T}_{2} \frac{h^{2}}{12}\right) c \rho-\lambda_{2}\{\left.\frac{r^{\prime}}{r}\left(T_{0}^{\prime}+T_{2}^{\prime} \frac{h^{2}}{12}\right)+T_{0}^{\prime \prime}+T_{2}^{\prime \prime} \frac{h^{2}}{12}\right\} \\
&-\lambda_{1} \frac{1}{r^{2}}\left(\nabla_{0}+\frac{\nabla \nabla}{T_{2}} \frac{h^{2}}{12}\right)-\frac{q_{a}-q_{i}}{h}-\frac{Q_{0}}{h}=0 ; \\
& \dot{T}_{1} c \rho-\lambda_{2}\left\{\frac{r^{\prime}}{r} T_{1}^{\prime}+T_{1}^{\prime \prime}\right\}-\lambda_{2} \frac{1}{r^{2}} T_{1}-\frac{6\left(q_{a}-q_{i}\right)}{h^{2}}+\frac{12 \lambda_{3}}{h^{2}} T_{1}-\frac{12 Q_{1}}{h^{3}}=0 ; \\
&\left(\dot{T}_{0}+\dot{T}_{2} \frac{3 h^{2}}{20}\right) c \rho-\lambda_{2}\left\{\frac{r^{\prime}}{r}\left(T_{0}^{\prime}+T_{2}^{\prime} \frac{3 h^{2}}{20}\right)+T_{0}^{\prime \prime}+T_{2}^{\prime \prime} \frac{3 h^{2}}{20}\right\} \\
&-\lambda_{1} \frac{1}{r^{2}}\left(\nabla_{0}+T_{2} \frac{\nabla h^{2}}{20}\right)-\frac{3\left(q_{a}-q_{i}\right)}{h}+4 \lambda_{3} T_{2}-\frac{12 Q_{2}}{h^{3}}=0
\end{aligned}
$$

where $\partial / \partial t=(\dot{)}) ; \partial / \partial \xi=\left(^{\nabla}\right) ; \partial / \partial \psi=()^{\prime} ;$ and $Q_{n}=\int_{-h / 2}^{h / 2} \eta(\zeta)^{n} d \zeta$ with $n=0,1,2$ as integrated internal heat generation.

A similar approach was proposed by Takezono et al. [7] using the finite difference method in space and time to determine the transient thermal field. In ASTRA, the stationary problem is solved similarly to the elastic approach as described above (see subsection 2.2). The three differential equations (Eqs. (6)(8)) are transferred into ordinary differential equations using Fourier series in the circumferential direction and summarized in a differential matrix. This differential matrix is integrated using numerical integration in the meridional direction. With this, the thermal conductivity matrix is computed (see Eq. (1)) 
for each element. Further computations are performed using standard finite element method. To obtain the transient solution of the thermal problem, Eq. (1) is solved using the Crank-Nicolson time integration method. Due to the high heat fluxes in a rocket nozzle and, consequently, the high temperatures, the radiation losses have to be considered to get meaningful results. An iteration procedure is implemented to consider the loss of energy to the environment due to radiation if needed. The energy flux caused by radiation is computed using the Stefan-Boltzmann law: $q_{\mathrm{rad}}=\varepsilon \sigma \theta_{\text {surf }}^{4}$ where $\varepsilon$ is the overall emissivity; $\sigma$ is the Stefan-Boltzmann constant; and $\theta_{\text {surf }}$ is the surface temperature.

Using the stationary or the transient thermal field the elastic deformation of the shell can be computed with the linear static, nonlinear static, or the dynamic solver.

\subsection{Aero-Thermoelastic Coupling Module}

The partitioned approach followed in the aerothermoelastic solution scheme makes necessary the spatial coupling of the single-field solvers involved as well as their synchronization. This is the primary task of the ATCM. For the temporal coupling in steady or unsteady simulations, several strong and loose schemes are available [8]. In order to reduce the computational costs, several predictorcorrector schemes are implemented for the solution process of unsteady problems. In the predictor step, either the structural deformation is directly extrapolated or calculated from extrapolated aerodynamic loads. In the corrector steps, the structural solution for the next time step is repeated with the loads computed by the flow solver instead of the extrapolated ones. With focus on numerical stability, computational efficiency and accuracy, very good results in comparison with strongly coupled computations could be achieved applying these schemes. In the presented results, an underrelaxed Gauss-Seidel scheme is applied since the study is focused on steady test cases. This scheme leads to an iterative execution of one single-field solver by holding the results of the other as constant.

The spatial coupling involves the transfer of mechanical loads and deformations as well as heat fluxes and temperatures between the surface grid of the considered configuration in the flow solver and the structural grid. In order to be valid from the physical point of view, any projection scheme has to be conservative with the following two criteria. First of all, the total force and moment vectors as well as the heat fluxes must be preserved during the projection. Second, during steady simulations, the elastic strain energy of the structure must be identical to the work performed by the aerodynamic loads on the wetted surface. During unsteady simulations, also the instantaneous power exchanged over the coupling surface must be the same on both sides. From the flow solver and the volume mesh deformation code, further numerical requirements arise affecting the projection of deformations from the structure back to the wetted surface. 
In particular, the deformed surface mesh should be smooth in order to assure good convergence of the flow solution. Several projection algorithms are available inside the ATCM: the Finite Interpolation Element (FIE) method [8-11] is an uncomplicated method that uses the shape functions of the structural model to divide aerodynamic surface loads among the nodes of the closest structural element. The Global Spline-Based (GSB) [12] and the Moving-Least-Squares (MLS) [13] methods constitute further alternatives suitable for reduced structural models. Both determine a function approximation to the nodal displacement distribution and evaluate it at the surface points. The two methods differ primarily in their choice of interpolation function. The GSB method derives the nodal values with one linear or quadratic polynomial and additional contributions of radial basis functions around the support points. Instead, the MLS method uses local interpolation functions exclusively. All projection methods inside the ATCM operate on extensive quantities, i. e., aerodynamic forces as opposed to surface stresses, and heat fluxes $Q$ as opposed to heat flux densities $q$. This makes the ATCM independent of the formulation of the flow solver.

\subsubsection{Finite Interpolation Elements}

The established spatial coupling employed in the ACM/ATCM relies on the FIE method described in [11]. To transfer the heat flux from a load incidence point on the wetted surface to the structure, first the projection of this point onto the closest nearby structural element is determined (Fig. 4). The interpolation of the heat flux to equivalent heat fluxes at the finite-element (FE) nodes of the respective element is done by using the $\mathrm{FE}$ shape functions $h_{\theta}^{i}$. Then, the interpolation coefficients are defined by evaluating these shape functions at the natural coordinates $\xi_{\text {proj }}$ and $\eta_{\text {proj }}$ of the projection point. To assure that no artificial energy is created during the aerothermal coupling, the temperature and the heat flux of both sides of the interface have to be the same. In analogy to

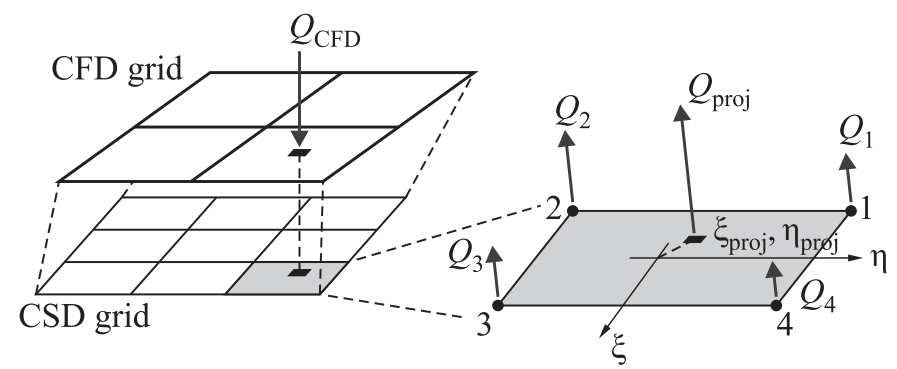

Figure 4 Transfer of heat fluxes (CSD - computational structural dynamics) 
the principle of virtual work for the structure, the functional of the thermal conduction problem is derived by the method of variation:

$$
\begin{aligned}
\Pi=\int_{V} \frac{1}{2}\left\{\lambda_{x}\left(\frac{\partial \theta}{\partial x}\right)^{2}+\lambda_{y}\left(\frac{\partial \theta}{\partial y}\right)^{2}+\lambda_{z}\left(\frac{\partial \theta}{\partial z}\right)^{2}\right\} & d V-\int_{V} \theta q^{B} d V \\
& -\int_{S} \theta^{S} q^{S} d S-\sum_{i} \theta^{i} Q^{i} .
\end{aligned}
$$

Here, $q^{B}$ is the heat flux created in the volume, and $q^{S}$ is the heat flux entering the volume through the surface. The variable $\lambda$ is the thermal conduction coefficient, the subscripts indicate its components in the different space dimensions. Right now, only air is considered; thus, all components of $\lambda$ are the same. $Q^{i}$ denotes the concentrated heat flux. It is assumed that the heat flux and temperature are constant over a boundary face $\Delta S^{i}$ of the grid of the flow solver. Therefore, all contributions of the heat flux are summed in the concentrated heat flux which is computed as follows: $Q^{i}=-\lambda \nabla \theta \Delta S^{i}$. To guarantee a conservative coupling, the potential $\Pi$ on both sides of the coupling interface has to be the same:

$$
\Pi_{\mathrm{CFD}}=Q_{\mathrm{CFD}} \delta \theta_{\mathrm{CFD}}=\Pi_{\mathrm{CSD}}=\sum_{i} Q_{\mathrm{CSD}}^{i} \delta \theta_{\mathrm{CSD}}^{i} .
$$

The temperature $\theta_{\mathrm{CFD}}$ can be expressed using the temperatures $\theta_{\mathrm{CSD}}^{i}$ at the nodes of the structural grid:

$$
\theta_{\mathrm{CFD}}=\sum_{i} h_{\theta}^{i}\left(\xi_{\mathrm{proj}}, \eta_{\mathrm{proj}}\right) \theta_{\mathrm{CSD}}^{i}
$$

Combining Eqs. (9) and (10) yields:

$$
Q_{\mathrm{CFD}} \sum_{i} h_{\theta}^{i}\left(\xi_{\mathrm{proj}}, \eta_{\mathrm{proj}}\right) \delta \theta_{\mathrm{CSD}}^{i}=\sum_{i} Q_{\mathrm{CSD}}^{i} \delta \theta_{\mathrm{CSD}}^{i}
$$

The heat flux at the nodes of the structural grid can then be described by

$$
Q_{\mathrm{CSD}}^{i}=h_{\theta}^{i}\left(\xi_{\mathrm{proj}}, \eta_{\mathrm{proj}}\right) Q_{\mathrm{CFD}} .
$$

To ensure conservation, the same procedure has to be followed in the backward direction when projecting the structural temperature distribution to the wetted surface. It is interpolated from the structure nodal values using the shape function values at the same projection points as before. Generally, the Finite Interpolation Method is grid independent and has no constrains on the element types on the aerodymamic or structural surface. Nevertheless, to avoid unphysical local effects, the area of the elements on the structural side has to be at least of the order of the area of the elements on the aerodynamic side. Otherwise, weighting functions can remedy unphysical errors [8]. 


\subsubsection{Moving-Least-Squares Interpolation}

The MLS interpolation method was first applied to spatial coupling in aeroelasticity by Quaranta et al. [13]. The projection is accomplished by performing an MLS interpolation about each load incidence point $\vec{x}_{n}$ with $N_{\delta}$ structural nodes inside of the support radius $\delta$ as supports. The projection quantity is approximated with low-order polynomials with $Q$ monomials. The monomial vectors are either

$$
\vec{m}=(1, x, y, z)^{\mathrm{T}} \quad \text { or } \quad \vec{m}=\left(1, x, y, z, x^{2}, y^{2}, z^{2}, x y, y z, z x\right)^{\mathrm{T}} .
$$

The coefficients $\boldsymbol{\alpha}(\overrightarrow{\vec{x}})$ at each interpolation support point result from an MLS fit with Wendland radial basis functions (RBFs) [14] as weights. For every surface point $\overrightarrow{\hat{x}}$ and its $N_{\delta}$ support points inside the support radius $\delta$, a functional

$$
I\left(\overrightarrow{\hat{x}}, \vec{x}_{n}\right)=\int_{\Omega_{\delta}} \phi\left(\overrightarrow{\hat{x}}, \vec{x}_{n}\right)\left(\vec{m}^{T}\left(\vec{x}_{n}\right) \boldsymbol{\alpha}\left(\vec{x}_{n}\right)-u_{\lambda}\left(\vec{x}_{n}\right)\right)^{2} d \Omega\left(\vec{x}_{n}\right)
$$

has to be minimized for the coefficients $\boldsymbol{\alpha}\left(\vec{x}_{n}\right)$. For the aerothermalelastic coupling with

$$
u_{\lambda} \in\left(u_{x}, u_{y}, u_{z}, \theta\right),
$$

the discrete form of this functional is reduced to the normal equation through a variation of coefficients $\delta \boldsymbol{\alpha}$ :

$$
\underbrace{\left[\vec{m}\left(\vec{x}_{n}\right)\right] \Phi\left(\overrightarrow{\hat{x}}, \vec{x}_{n}\right)\left[\vec{m}\left(\vec{x}_{n}\right)\right]^{\mathrm{T}}}_{A} \boldsymbol{\alpha}\left(\vec{x}_{n}\right)=\underbrace{\left[\vec{m}\left(\vec{x}_{n}\right)\right] \Phi\left(\overrightarrow{\hat{x}}, \vec{x}_{n}\right)}_{B}\left\{u_{\lambda}\left(\vec{x}_{n}\right)\right\} .
$$

Herein, $\Phi\left(\overrightarrow{\hat{x}}, \overrightarrow{\vec{x}}_{n}\right)=E\left\{\phi\left(\overrightarrow{\hat{x}}, \vec{x}_{n}\right)\right\}$ is the diagonal matrix of RBF weighting factors. Inserting the polynomial expression of the interpolation function $\vec{m}^{\mathrm{T}}(\overrightarrow{\hat{x}})\left[\boldsymbol{\alpha}\left(\overrightarrow{\vec{x}}_{n}\right)\right]$ yields

$$
u_{\lambda}(\overrightarrow{\hat{x}})=\underbrace{\vec{m}^{\mathrm{T}}(\overrightarrow{\hat{x}}) A^{-1} B}_{\vec{P}(\overrightarrow{\hat{x}})}\left\{u_{\lambda}\left(\vec{x}_{n}\right)\right\} .
$$

The row matrix $\vec{P}(\overrightarrow{\hat{x}})$ describes the projection between a single surface point $\overrightarrow{\hat{x}}$ and the $N_{\delta}$ support points inside the support radius. In the MLS method, the projection matrix is built row by row for each surface point separately. The final projection matrix $P$ is assembled from the $M$ row matrices of all surface points. For each surface point, a $Q \times Q$-matrix $A$ has to be set up and inverted. Its condition number and, thus, its invertability depends on the number of support points and their spatial arrangement. Practical experience has revealed that the regularization of the linear systems of Eqs. (11) by left multiplication of 
$\left[\vec{m}\left(\vec{x}_{n}\right)\right]$ is highly detrimental to its condition number. A more accurate and robust numerical solution can be achieved if instead, for each surface point, the $N_{\delta}$ overdetermined systems of equations

$$
\Phi\left(\overrightarrow{\hat{x}}, \vec{x}_{n}\right)\left[\vec{m}\left(\vec{x}_{n}\right)\right]^{\mathrm{T}}\left[\boldsymbol{\alpha}\left(\vec{x}_{n}\right)\right]=\Phi
$$

are solved with $Q R$ decomposition, yielding $N_{\delta}$ tuples of polynomial coefficients $\boldsymbol{\alpha}^{*}\left(\overrightarrow{\vec{x}}_{n}\right)$ for unit deflections or heat fluxes $u_{\lambda}^{*}\left(\vec{x}_{n}\right)$. The final row entry to the projection matrix then is

$$
\vec{P}(\overrightarrow{\hat{x}})=\vec{m}^{\mathrm{T}}(\overrightarrow{\hat{x}})\left[\boldsymbol{\alpha}^{*}\left(\vec{x}_{n}\right)\right] .
$$

Because the MLS interpolation method has only local support, the projection matrix is sparse, greatly reducing memory requirements.

\section{VALIDATION}

Each of the individual solvers has been compared to the experimental measurements or to the numerical results. Simulations concerned with supersonic boundary layer flow [15] and simulations of an oblique shock impinging on a boundary layer [16] showed a good agreement between numerical results and experimental measurements. The analysis methods of the structural solver ASTRA necessary for the aerothermoelastic coupling, like the dynamic analysis, the thermal and the thermoelastic analyses, are validated using commercial FE-codes. For this purpose, computations with full 3D models are performed using Abaqus and compared to the equivalent ASTRA computations.

The current research concentrates on the validation of the fully coupled solver for aerothermoelastic problems. First, the aeroelastic and aerothermal coupling between QUADFLOW and ASTRA are considered separately. Quite good results are required for the validation of the fully coupled aerothermolelastic solver. Here, the validation of the thermoelastic module of ASTRA against Abaqus and the validation of the aeroelastic coupling chain with experimental data provided by the DLR Cologne are presented.

\subsection{Validation of the Aeroelastic Coupling}

\subsubsection{Experimental setup}

The experimental setup is designed to investigate the interaction between an oblique impinging shock and an elastic panel at supersonic inflow conditions; for a detailed description please refer [17] .

The conditions proposed for the experiment are summarized in Table 1. The main constructional elements of the experimental setup are a 20 degree wedge 
Table 1 Flow conditions proposed for the experiments

\begin{tabular}{cccrcc}
\hline $\mathrm{M}$ & $T_{\text {tot }}, \mathrm{K}$ & \multicolumn{1}{c}{$p_{\text {tot }}$, bar } & $T_{\infty}, \mathrm{K}$ & $p_{\infty}$, bar & Re \\
\hline 2.5 & 287.4 & 3.67 & 128.0 & 0.22 & $36.7 \cdot 10^{6}$ \\
3.0 & 286.0 & 5.85 & 102.4 & 0.16 & $45.6 \cdot 10^{6}$ \\
3.5 & 284.8 & 9.16 & 82.8 & 0.12 & $55.8 \cdot 10^{6}$ \\
4.0 & 288.1 & 13.75 & 68.6 & 0.09 & $64.4 \cdot 10^{6}$ \\
\hline
\end{tabular}

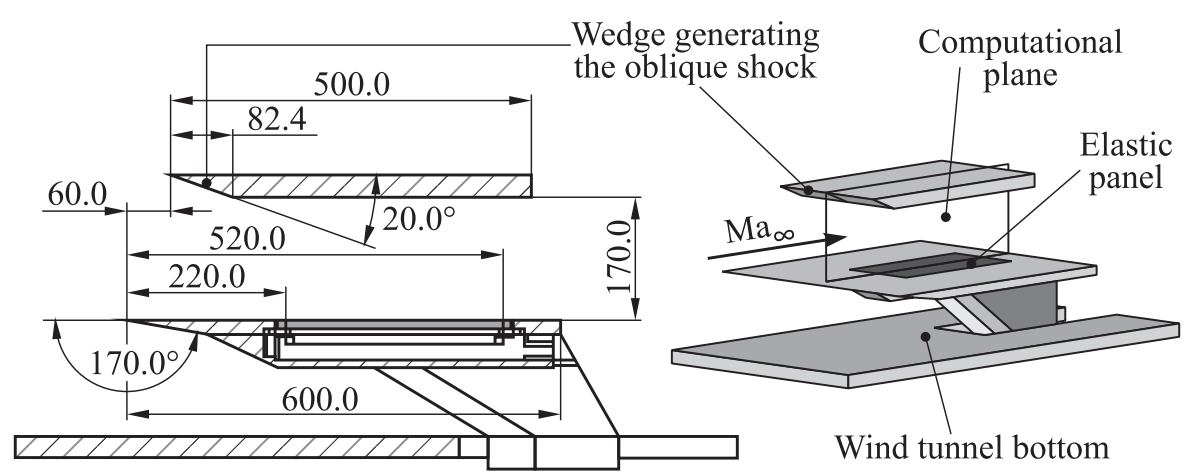

Figure 5 Dimensions and perspective illustration of the experimental setup. Dimensions are in millimeters

and an elastic panel. The wedge is positioned at the beginning of the upper part of the flow channel. The elastic panel is embedded in the lower part of this channel. It is positioned such that the oblique shock being generated from the wedge at supersonic flow conditions impinges on the panel at about one third of its length. Over the length of the panel, the channel between wedge and panel has a constant cross section.

Figure 5 illustrates the experimental setup. The elastic panel has a width of $200 \mathrm{~mm}$, a length of $300 \mathrm{~mm}$, and is clamped on both sides over $15 \mathrm{~mm}$ of additional length. The table holding the panel is as wide and long as the wind tunnel is wide, i.e., $600 \mathrm{~mm}$. Alongside the panel, an elastic sealing is installed which prevents pressure compensation. The DLR Cologne will conduct the experiments in its Trisonic Wind Tunnel (TMK). Since that tunnel has a cross-sectional area of $600 \times 600 \mathrm{~mm}$, the tunnel walls are sufficiently far away from the center plane of the panel.

\subsubsection{Computational setup}

Based on the assumption that wind tunnel wall influences are insignificant, the computations are carried out at first purely in two dimensions. The computa- 


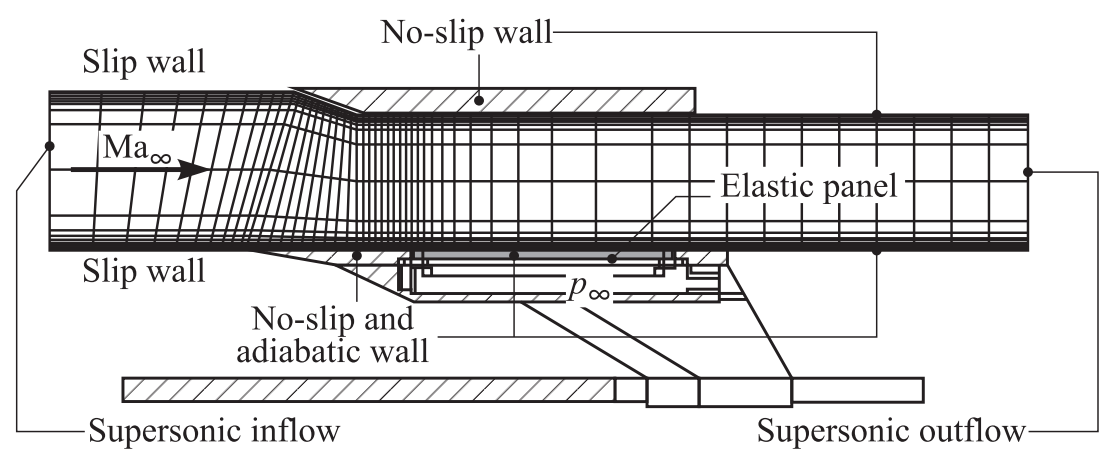

Figure 6 Computational grid (initial nonadapted grid) and imposed flow boundary conditions
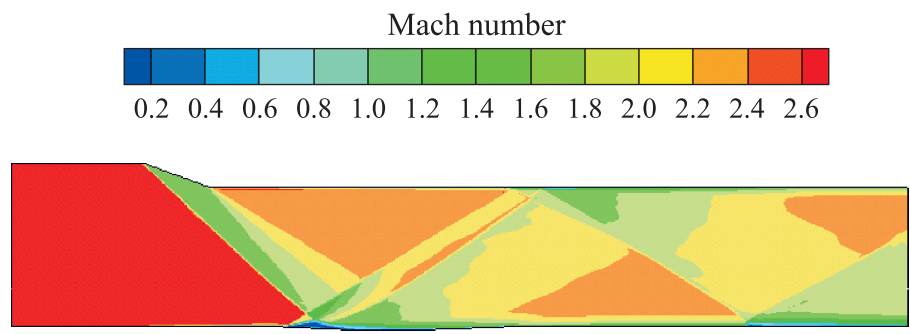

Figure 7 Flow field for $\mathrm{M}=2.5$.

tional domain and the boundary conditions imposed on the domain boundaries are shown in Fig. 6 .

The flow is assumed to be fully turbulent. This is justified $(i)$ due to the high Reynolds number (see Table 1); and (ii) because a tripping device is to be placed at the leading edge of the table which will enforce laminar-turbulent transition. The computed flow field for the hightest load case $(\mathrm{M}=2.5)$ is given in Fig. 7. In the experiment, the panel will be clamped on both sides. The following properties are used for the panel material: $E=2.06 \cdot 10^{11} \mathrm{~N} / \mathrm{m}^{2}$ and $G=0.78 \cdot 10^{11} \mathrm{~N} / \mathrm{m}^{2}$. The panel thickness in the numerical investigations is set to $2 \mathrm{~mm}$. It is discretized by 100 elements. To approximate the flat planel geometry, axisymmetric elements with very large radius are used. The pressure beneath the panel is applied equal to the inflow pressure which is 0.16 bar.

\subsubsection{Results}

For all conditions given in Table 1 aeroelastic computations using QUADFLOW and ASTRA coupled via ATCM are performed. The measured deformations of 


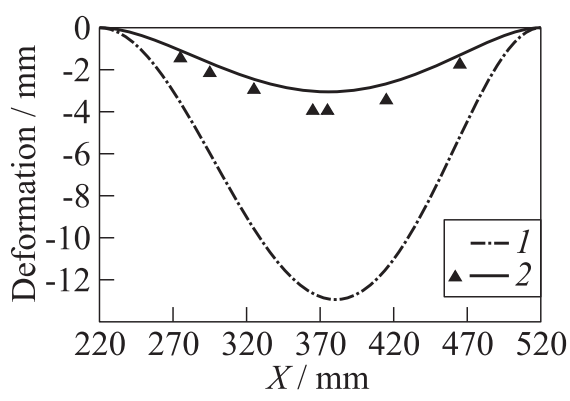

(a)

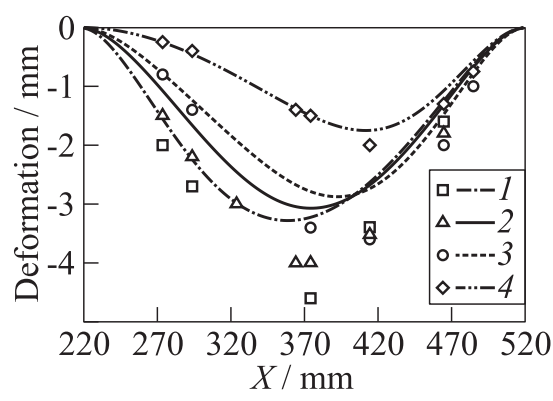

(b)

Figure 8 Comparisons of aeroelastic results using linear (FEAFA - 1) and nonlinear (ASTRA - 2) structural solvers $(a)$ and of numerical results (curves) using ASTRA with experimental data (signs) $(b): 1-\mathrm{M}=2.5 ; 2-3.0 ; 3-3.5$; and $4-\mathrm{M}=4.0$

the elastic panel are compared to the numerical results. The computational time exceeded the time of a CFD simulation only insignificantly thanks to the fast computations of the applied structural models as well as the spatial coupling methods.

The first approach using the linear solver FEAFA [8] showed large differences to the experiments. By using the nonlinear structural solver ASTRA a much better fit between measured data and computed deformations could be achieved. The comparison between the linear and nonlinear solver for $\mathrm{M}=3.0$ is shown in Fig. $8 a$.

The comparison between the experimental deformations and the displacements computed with ASTRA for all considered cases is summarized in Fig. $8 b$. Caused by the movement of the shock to the end of the elastic panel, the deformation decreased with increasing Mach numbers. For the deformations induced by the flow, the numerical results of the aeroelastic computations underestimate the measured data. The deviation increased for lower Mach numbers.

The difference can be explained by the clamped boundary conditions used for the simulations. In reality, the frame to which the elastic panel is riveted deforms during the experiment [18]. To approximate the behavior of the elastic boundary conditions, they are modeled in a way which can be described as a set of springs. One spring is meant to hinder the in-plane deformation (spring constant $k_{\text {lin }}=1.25 \cdot 10^{9} \mathrm{~N} / \mathrm{m}$ ) and a second rotational spring is meant to resist the rotation around the edge of the panel (spring constant $k_{\text {rot }}=1667 \mathrm{~N}$ ). The spring constants were calibrated using the results of experiments during which the elastic panel was exposed to a constant pressure difference between the upper and the lower sides (Fig. 9).

The results of the aeroelastic computations using the elastic boundary conditions are summarized in Fig. 10. For the three lowest initial flow velocities 


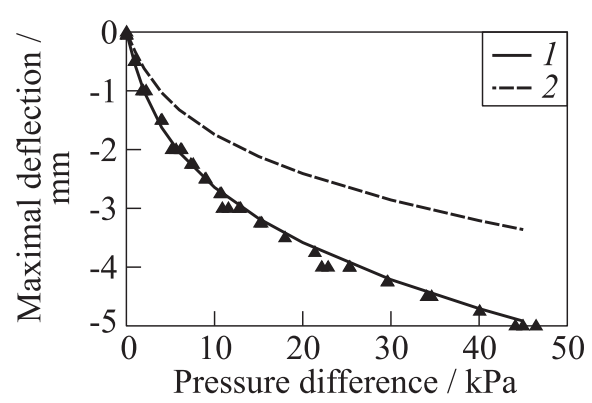

Figure 9 Calibration of spring constants: 1 - elastic and 2 - clamped boundary conditions (ASTRA). Signs refer to experiments

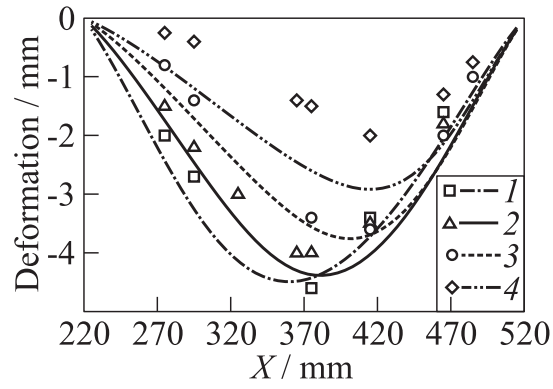

Figure 10 Comparison of numerical results with elastic boundary conditions using ASTRA (CFD-CMS, curves) with experimental data (signs): $1-\mathrm{M}$ $=2.5 ; 2-3.0 ; 3-3.5 ;$ and $4-$ $\mathrm{M}=4.0$

$(\mathrm{M}=2.5,3$, and 3.5), the deformation lies close to the experimental data, which leads to a better prediction of the deformation compared to in the simulations using clamped boundary conditions (see Fig. 8b).

\subsection{Validation of ASTRA}

The thermoelastic module of ASTRA is validated using several code-to-code comparison computations with the commercial solver Abaqus. One simple example is presented to compare the numerical results. A nozzle-like axisymmetric shell (Fig. 11) is clamped on one side $(x=0)$ and also, a thermal boundary condition is imposed on the opposite edge $(x=L$ and $T_{L}=0{ }^{\circ} \mathrm{C}$ ). The material of the structure is $\mathrm{C} / \mathrm{C}-\mathrm{SiC}$. All necessary material and geometric parameters are as follows: $R_{1}=200 \mathrm{~mm}, R_{2}$ $=400 \mathrm{~mm} ; L=600 \mathrm{~mm} ; h=4 \mathrm{~mm}$; $E=6 \cdot 10^{10} \mathrm{~N} / \mathrm{m}^{2} ; G=1.4 \cdot 10^{10} \mathrm{~N} / \mathrm{m}^{2} ;$

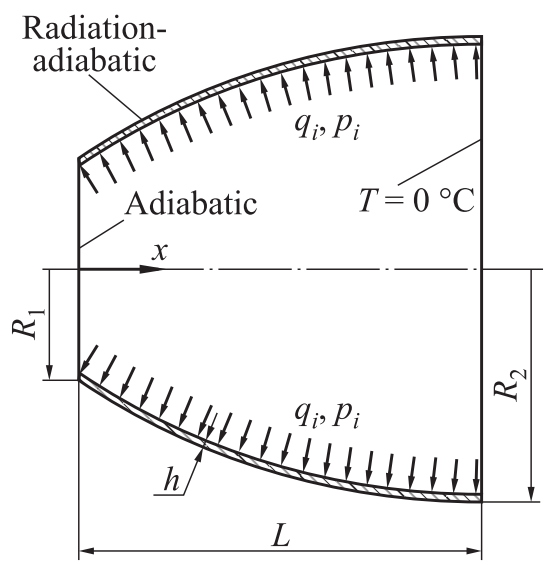

Figure 11 Nozzle-like structure for code-to-code comparison $\nu=0.05 ; \alpha=2.5 \cdot 10^{-6} 1 / \mathrm{K} ; \rho$ $=1870 \mathrm{~kg} / \mathrm{m}^{3} ; \lambda_{\text {inplane }}=25 \mathrm{~W} /(\mathrm{mK}) ; \lambda_{\text {outofplane }}=15 \mathrm{~W} /(\mathrm{mK}) ;$ and $c$ $=748 \mathrm{~J} /(\mathrm{kg} \cdot \mathrm{K})$. The structure is loaded with a constant thermal flux of $q_{i}$ 


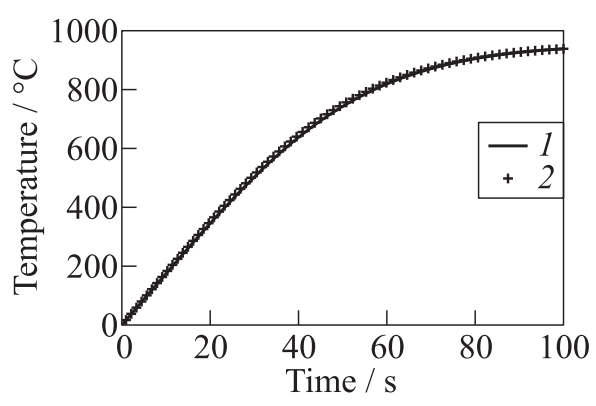

Figure 12 Transient temperature at $x=0: 1-$ Abaqus and $2-$ ASTRA

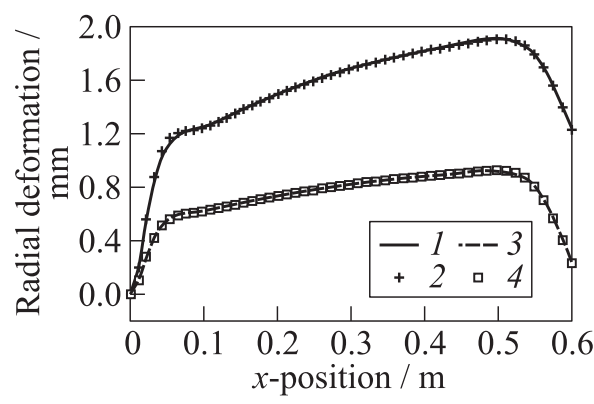

Figure 13 Deformation after $100 \mathrm{~s}: 1$ Abaqus; 2 - ASTRA; 3 - Abaqus without pressure; and $4-$ ASTRA without pressure

$=100000 \mathrm{~W} / \mathrm{m}^{2}$ on the inner surface. Also, on the inner surface, a constant inner pressure of $p_{i}=15 \cdot 10^{6} \mathrm{~N} / \mathrm{m}^{2}$ is applied.

In ASTRA, the structure is idealized with 49 axisymmetric elements or 150 thermal and 200 mechanical degrees of freedom. The Abaqus model, on the other hand, is fully 3D and is made of 4-node shell elements with 6 integration points through the shell thickness for the thermal computation. This model has 3570 nodes or 21420 thermal degrees of freedom and the same number of mechanical degrees of freedom.

Transient comparison computations were performed, considering thermal radiation to the ambient. In Fig. 12, the temperature at the left edge $(x=0)$ of the structure is plotted vs. time. Both solvers show almost identical results. Similar agreement is computed for the deformations. In Fig. 13, the deformation is shown due to the combination of thermal and pressure loads after 100-second heating. ASTRA as well as Abaqus compute the deformation after the temperature field is determinated.

\section{CONCLUDING REMARKS AND OUTLOOK}

A partitioned coupling approach towards an aerothermoelastic solver using reduced structural models is presented. Following this approach, the CFD solver QUADFLOW is coupled with the structural solver ASTRA to simulate aeroelastic problems. The required methods for the information exchange between both single field solvers, which are implemented in the ATCM, were presented. The computational costs of the presented steady-coupled simulations applying reduced structural models exceeds the cost of CFD simulations insignificantly. First results of the modular aeroelastic solver show good agreement with the 
experimental data. As preparation for aerothermoelastic simulations ASTRA was extended by a thermoelastic module, which produces essentially the same results compared with the commercial solver Abaqus.

After the successful validation of the thermal branch of ASTRA and the aeroelastic coupling between QUADFLOW and ASTRA, the validation of the aerothermal coupling will be performed. It will be based on the experiments which are conducted at the DLR Cologne. Here, air with a free stream temperature $T \approx 500 \mathrm{~K}$ and a free stream Mach number Ma $\approx 7.5$ will flow over a ceramic structure. The final validation of the aerothermoelastic coupling will be based on the experiments conducted in the IMENS project [19]. This project was concerned with the investigation of thermal and mechanical fluid-structure interactions of spacecraft vehicles. A generic flap model had been manufactured and investigated in the arc-heated facility L3K at DLR Cologne.

\section{ACKNOWLEDGMENTS}

The funding of the work by the German Research Foundation within the framework of the Collaborative Research Center SFB/TRR 40 "Technological foundations for the design of thermally and mechanically highly loaded components of future space transportation systems" is gratefully acknowledged. Computing resources were provided by the RWTH Aachen University Center for Computing and Communication and supported by the German Research Foundation under GSC 111 (AICES). The authors are also grateful to DLR Cologne for producing the experimental data required for the validation process.

\section{REFERENCES}

1. Ballmann, J. 2003. Flow modulation and fluid-structure interaction at airplane wings. Numerical notes on fluid mechanics and multidisciplinary design. Springer Verlag. 84.

2. Müller, S. 2002. Adaptive multiscale schemes for conservation laws. Lecture notes on computational science and engineering. Springer Verlag. 27.

3. Lamby, Ph. 2007. Parametric multi-block grid generation and application to adaptive flow simulations. Doctoral Thesis. RWTH Aachen University.

4. Rittweger, A. 1992. Statik, Stabilität und Eigenschwingungen anisotroper Rotationsschalen beliebigen Meridians mit der Übertragungsmatrizen-Methode. Doctoral Thesis. RWTH Aachen University.

5. Rittweger, A., T. Scherman, H.-G. Reimerdes, and H. Öry. 1995. Influence of geometric imperfections on the load capacity of orthotropic stiffened and composite shells of revolution with arbitrary meridians and boundary conditions. Thin-Walled Structures. 23:237-54. 
6. Öry, H., H.-G. Reimerdes, T. Schmid, A. Rittweger, and J. Gomez-Garcia. 2002. Imperfection sensitivity of an orthotropic spherical shell under external pressure. Int. J. Non-Linear Mech. 37:669-89.

7. Takezono, S., K. Tao, T. Aoki, and E. Inamura. 1995. Elasto/visco-plastic deformation of shells of revolution under thermal loading due to fluid. JSME Int. J. 38:185-93.

8. Reimer, L., C. Braun, G. Wellmer, M. Behr, and J. Ballmann. 1997-2008. Development of a modular method for computational aero-structural analysis of aircraft. In: Summary of flow modulation and fluid-structure interaction findings - results of the Collaborative Research Center SFB 401 at the RWTH Aachen University. Ed. W. Schröder. Aachen, Germany: Springer Verlag.

9. Beckert, A. 2000. Coupling fluid (CFD) and structural (FE) models using finite interpolation elements. Aerosp. Sci. Technol. 4:13-22.

10. Boucke. A. 2003. Kopplungswerkzeuge für aeroelastische Simulationen. Doctoral Thesis. RWTH Aachen University.

11. Braun, C. 2007. Ein modulares Verfahren für die numerische aeroelastische Analyse von Luftfahrzeugen. Doctoral Thesis. RWTH Aachen University.

12. Beckert, A., and H. Wendland. 2001. Multivariate interpolation for fluid-structureinteraction problems using radial basis functions. Aerospace Sci. Technol. 5(2):12534 .

13. Quaranta, G., P. Masarati, and P. Mantegazza. 2005. A conservative mesh-free approach for fluid-structure interface problems. Conference (International) on Computational Methods for Coupled Problems in Science and Engineering. Barcelona, Spain.

14. Wendland, H. 1995. Piecewise polynomial, positive definite and compactly supported radial functions of minimal degree. Adv. Comput. Math. 4:389-96.

15. Fernholz, H. H., and P. J. Finley. 1980. A critical compilation of compressible turbulent boundary layer data. AGARD-AG-223.

16. Dupont, P. C. Haddad, and J. F. Debieve. 2006. Space and time organization in a shock-induced separated boundary layer. J. Fluid Mech. 559:255-77.

17. Willems, S., B. Esser, and A. Gülhan. 2009. Designing an experimental set-up for research into supersonic flow-structure interaction. In: Annual report of the SFB/TRR40. Eds. N. Adams, B. Weigand, R. Radespiel, T. Sattelmayer, and W. Schröder. 297-396.

18. Willems, S., A. Gülhan, and B. Esser. 2012. Shock-induced fluid-structure interaction on flexible wall in supersonic turbulent flow. In: Progress in flight physics. Eds. Ph. Reijasse, D. Knight, M. I. Ivanov, and I. Lipatov. Advances in aerospace sciences EUCASS ser. EDP Sciences-TORUS PRESS. Vol. 5.

19. Esser, B., A. Gülhan, R. Schäfer, and A. Mack. 2003. Experimental investigation of thermomechanical fluid-structure interaction in high-enthalpy flow. 5th Congress (International) on Thermal Stresses and Related Topics. Blacksburg, Virginia, USA. 